\title{
Replacement of the Thiosugar of Auranofin with lodide Enhances the Anticancer Potency in a Mouse Model of Ovarian Cancer
}

\author{
Tiziano Marzo, ${ }^{\dagger, \nabla} \nabla_{\odot}$ Lara Massai, ${ }^{\ddagger, \nabla}$ Alessandro Pratesi, ${ }^{\ddagger \odot}$ Matteo Stefanini, ${ }^{\S}$ Damiano Cirri,
} Francesca Magherini, ${ }^{\| \odot}$ Matteo Becatti, ${ }^{\|}$Ida Landini, ${ }^{\perp}$ Stefania Nobili, ${ }^{\perp}$ Enrico Mini, ${ }^{\perp}$ Olivia Crociani, ${ }^{\S}$

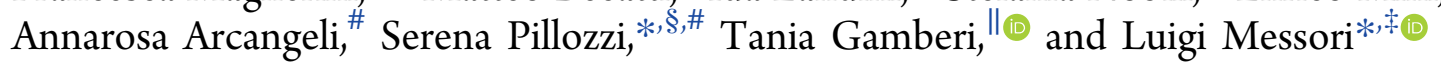

${ }^{\dagger}$ Department of Pharmacy, University of Pisa, via Bonanno Pisano 6, 56126 Pisa, Italy

${ }^{\ddagger}$ Laboratory of Metals in Medicine (MetMed), Department of Chemistry “U. Schiff”, University of Florence, via della Lastruccia 3, 50019 Sesto Fiorentino, Italy

${ }^{\S}$ DI.V.A.L. Toscana s.r.l., via Madonna del Piano 6, 50019 Sesto Fiorentino, Italy

"Department of Biochemical, Experimental and Clinical Sciences "Mario Serio", University of Florence, viale G.B. Morgagni 50, 50134 Firenze, Italy

${ }^{\perp}$ Department of Health Sciences, University of Florence, viale Pieraccini 6, 50139 Firenze, Italy

${ }^{\#}$ Department of Experimental and Clinical Medicine, University of Florence, viale G.B. Morgagni 50, 50134 Firenze, Italy

\section{Supporting Information}

ABSTRACT: In recent years, a few successful attempts were made to repurpose the clinically approved antiarthritic gold drug, Auranofin (AF), as an anticancer agent. The present study shows that the iodido(triethylphosphine)gold(I) complex, ( $\mathbf{E t}_{3} \mathbf{P A u I}$ hereafter) - an $\mathbf{A F}$ analogue where the thiosugar ligand is simply replaced by one iodide ligandmanifests a solution chemistry resembling that of AF and exerts similar cytotoxic and proapoptotic effects on A2780 human ovarian cancer cells in vitro. However, when evaluated in a preclinical orthotopic model of ovarian cancer, $\mathbf{E t}_{3} \mathbf{P A u I}$ produces a far superior anticancer action than AF inducing a nearly complete tumor remission. The highly promising in vivo performances here documented for $\mathrm{Et}_{3} \mathbf{P A u I}$ warrant its further evaluation as a drug candidate for ovarian cancer treatment.

KEYWORDS: Auranofin, gold compounds, ovarian cancer, metal-based drugs, in vivo orthotopic model

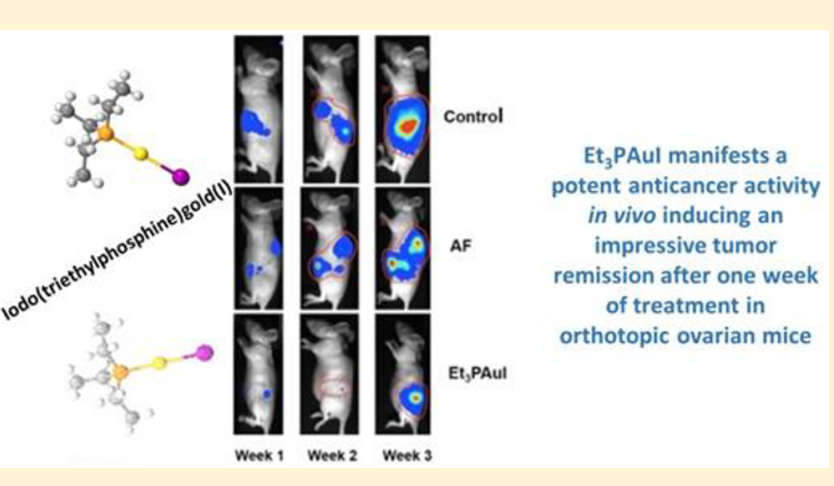

A uranofin, a linear gold(I) complex bearing triethylphosphine and thioglucose tetraacetate as ligands, is an orally administered drug, in clinical use since 1985 for the treatment of rheumatoid arthritis. AF is generally reputed as a reasonably safe drug for human use, even for long chronic treatments. ${ }^{1,2}$ Beyond its clinically established antiarthritic properties, in the frame of the so-called drug repurposing strategies, AF has been extensively tested in recent years as a potential antiparasitic, antimicrobial, and anticancer agent with encouraging results. $^{3-5}$ Of considerable interest are the antiproliferative properties of this compound that have been known since the early $1980 \mathrm{~s}^{5-7}$ and were later assessed in the NCI 60 cancer cell line panel and in a few in vivo models. ${ }^{8-10}$ On the ground of its anticancer properties, AF in the course of the last years has entered three distinct clinical trials, two of them (ovarian and lung cancer) still ongoing and one (leukemia) closed. ${ }^{11-13}$ Results of these trials have not been disclosed yet. At the same time, considerable evidence has been gathered that a few specific biomolecular targets such as the enzyme thioredoxin reductase, the proteasome system, the NF- $\kappa \mathrm{B}$ pathway and other transcription factors, are crucially involved in mediating the cellular effects of AF as well of other gold (I/III) compounds. $^{14-18}$

It has been hypothesized, and then demonstrated, that the biological and pharmacological properties of AF might be kept and hopefully improved/modulated by replacing the thiosugar ligand with other ligands such as various halides. In principle, replacement of the thiosugar ligand with halide ligands may lead to an increased lipophilicity and an enhanced drug bioavailability and biodistribution. However, this modification should not affect substantially the drug's pharmacological profile, as the invariant $\left[\mathrm{Et}_{3} \mathrm{PAu}^{+}\right]$moiety is assumed to be the

Special Issue: Highlighting Medicinal Chemistry in Italy

Received: January 7, 2019

Accepted: February 7, 2019

Published: February 7, 2019 
"true pharmacophore" whereas the thiosugar ligand is believed to act mainly as a carrier ligand. ${ }^{18}$ Accordingly, the actions of iodido(triethylphosphine)gold(I) complex, Et ${ }_{3} \mathbf{P A u I}$, and AF, have been comparatively analyzed toward ovarian cancer models both in vitro and in vivo. Et $_{3}$ PAuI is obtained by replacement of the thiosugar ligand with an iodide ligand according to the reported synthetic procedures ${ }^{18}$ (see Supporting Information for detailed synthesis and characterization).

The chemical structures of $\mathbf{A F}$ and $\mathbf{E t}_{3} \mathbf{P A u I}$ are shown in Figure 1.

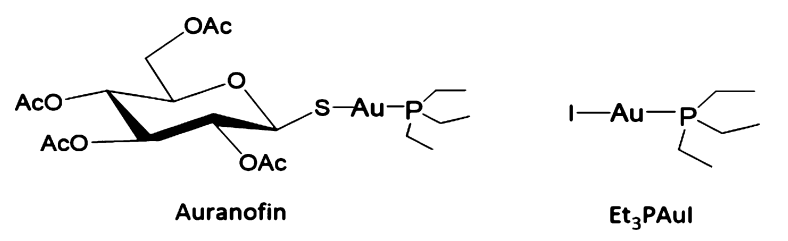

Figure 1. Chemical structures of Auranofin and iodido(triethylphosphine)gold(I).

Replacement of the thiosugar ligand with iodide results into a greatly increased lipophilic character (a $\log \mathrm{P}$ value of 4.6 has been measured by ICP-OES in the previous work ${ }^{18}$ ) with respect to both $\mathbf{E t}_{3} \mathbf{P A u C l}$ and $\mathbf{A F}$ (this latter compound has been previously described and used experimentally for rheumatoid arthritis treatment), ${ }^{19}$ for which $\log \mathrm{P}$ values of 1.7 and 1.6 were measured, respectively. ${ }^{18}$ In spite of that, $\mathrm{Et}_{3} \mathbf{P A u I}$ is still appreciably soluble in aqueous solutions, where it manifests a very high stability as documented by a previous systematic NMR analysis, carried out in different environments including biological media. ${ }^{18,20}$ As reported in the Supporting Information (see Figure S4), new ${ }^{31} \mathrm{P}$ NMR results clearly demonstrate a high stability of $\mathbf{E t}_{3} \mathbf{P A u I}$, with no changes in the structure of the molecule, even in the presence of a large excess of sodium chloride (up to $100 \mathrm{mM}$ ). The documented stability in physiological-like conditions makes the compound an ideal candidate for biological testing.

The antiproliferative properties of $\mathbf{E t}_{3} \mathbf{P A u I}$ compared to $\mathbf{A F}$ have been investigated in vitro toward A2780 human ovarian cancer cells. As displayed in Table 1, both compounds produce

Table 1. Inhibitory Effects of $\mathrm{AF}, \mathrm{Et}_{3} \mathrm{PAuI}$, and Cisplatin on A2780 Cells Following 72 h Drug Exposure As Determined by the Sulforhodamine B Assay ${ }^{a}$

\begin{tabular}{llll} 
& \multicolumn{3}{c}{$\mathrm{IC}_{50}(\mu \mathrm{M}) \pm \mathrm{SD}$} \\
\cline { 2 - 4 } cell lines & \multicolumn{1}{c}{ Et $\mathrm{Et}_{3}$ PAuI } & \multicolumn{1}{c}{ Auranofin } & \multicolumn{1}{c}{ cisplatin } \\
$\mathrm{A} 2780$ & $0.394 \pm 0.174$ & $0.754 \pm 0.139$ & $1.53 \pm 0.66$ \\
$n$ & 4 & 4 & 5 \\
$p^{b}$ & & 0.0138 &
\end{tabular}

${ }^{a}$ Four independent experiments were performed. SD, standard deviation; $n$, number of independent experiments. ${ }^{b} t$ test.

potent cytotoxic effects with $\mathrm{IC}_{50}$ values falling in the high nanomolar range. $\mathbf{E t}_{3} \mathbf{P A u I}$ is slightly more cytotoxic than $\mathbf{A F}$ and both result significantly more effective than cisplatin, the well-known anticancer metal compound. These results are consistent with those previously obtained in colon cancer cell lines for the same gold compounds and recently published literature ${ }^{18}$ further supporting the concept that the presence of the thiosugar ligand is not an essential requirement for the cytotoxic action and that substantial differences in the cellular uptake, despite the difference in the $\log \mathrm{P}$ value, between Et $_{3}$ PAuI and AF are unlikely to occur. Remarkably, in agreement with a previous observation, ${ }^{18}$ no appreciable cytotoxic effects were detected for $\mathbf{E t}_{3} \mathbf{P A u I}$ in normal cell lines, i.e., human fibroblasts (HDF cell line) and human embryonic kidney cells (HEK293) over the concentration range $0-5000 \mathrm{nM}$.

Then, we examined whether $\mathbf{E t}_{\mathbf{3}} \mathbf{P A u I}$ and $\mathbf{A F}$ are able to induce apoptosis in A2780 cells; accordingly, we performed flow cytometry analysis of annexin $\mathrm{V} /$ propidium iodide-stained A2780 cells, treated with these two gold compounds, at the respective $\mathrm{IC}_{50}$ values, for $72 \mathrm{~h}$. As shown in Figure $S 1$, the percentage of apoptotic cells in AF- and $\mathbf{E t}_{3}$ PAuI-treated samples is significantly increased (control vs AF, $p=0.0121$; control vs $\left.\mathbf{E t}_{3} \mathbf{P A u I}, p=0.0107\right)$. In order to evaluate and quantitate apoptosis via caspase activity, we checked the activity of caspase 3 by FAM-FLICA Caspase- 3 Assay Kit. The induction of apoptosis observed for $\mathrm{AF}$ and its iodide analogue may be related with the strong thioredoxin reductase inhibition potency. Indeed, the $\mathrm{IC}_{50}$ values for the inhibition of the enzyme are generally in nice agreement with cytotoxicity values. $^{18}$

The results collected evidenced that both gold compounds induce apoptosis through a caspase 3 activation, with AF being slightly more effective than $\mathbf{E t}_{3} \mathbf{P A u I}$ (control vs AF, $p=$ 0.0037; control vs $\mathbf{E t}_{3} \mathbf{P A u I}, p=0.04$, see Supporting Information).

$\mathbf{E t}_{3} \mathbf{P A u I}$ was subsequently evaluated in vivo in comparison to AF in two different ovarian cancer models, i.e., a xenograft subcutaneous and an orthotopic model. Preliminarily, drug tolerability studies were carried out to assess the in vivo safety of $\mathbf{E t}_{3} \mathbf{P A u I}$. The single-dose tolerability was evaluated in athymic nude mice over a 3-week observation period. $\mathbf{E t}_{3} \mathbf{P A u I}$ was administered by intraperitoneal (ip) injection using two different doses (20 and $40 \mathrm{mg} / \mathrm{kg}$ ). Only a single administration was performed, followed by a 3-week observation period; animals were controlled daily, and no signs of sufferings such as weight loss, mobility reduction and appetite reduction were registered. In addition, a pilot study for gold biodistribution has been carried out and a generalized higher accumulation for $\mathbf{E t}_{3} \mathbf{P A u I}$, both in organs and in the peripheral blood, was highlighted (see Supporting Information). Based on the above observations, we can hypothesize that the detected differences in the in vivo activity of the two compounds could be due to their different lipophilic character leading to an enhanced bioavailability and more favorable pharmacokinetics for $\mathbf{E t}_{\mathbf{3}} \mathbf{P A u I}$.

The xenograft model consisted of $1 \times 10^{6}$ A2780 cells implanted subcutaneously (sc) in both flanks of the animals. The growth of the tumor masses was measured daily with caliper and the treatment started when the volume of the masses reached $60 \mathrm{~mm}^{3}$. The treatment was performed by ip injections (three times a week for 2 weeks of treatment) of AF and $\mathbf{E t}_{3} \mathbf{P A u I}$, both $15 \mathrm{mg} / \mathrm{kg}$. The growth of the tumor masses was monitored over 21 days and time course is shown in Figure S3A,B respectively. At the end of the experiments, mice were sacrificed and the explanted masses measured; the volume $\left(\mathrm{cm}^{3}\right)$ was calculated using the ellipsoid equation. ${ }^{21}$ Remarkably, treatment with both $\mathbf{E t}_{3} \mathbf{P A u I}$ and $\mathrm{AF}$ was accompanied by a strong decrease of the tumor volume determined at the sacrifice (Figure 2, control: $2.96 \pm 0.06$ vs $\mathrm{Et}_{3}$ PAuI: $0.56 \pm 0.20, p<0.01$; control: $2.96 \pm 0.06$ vs AF: 


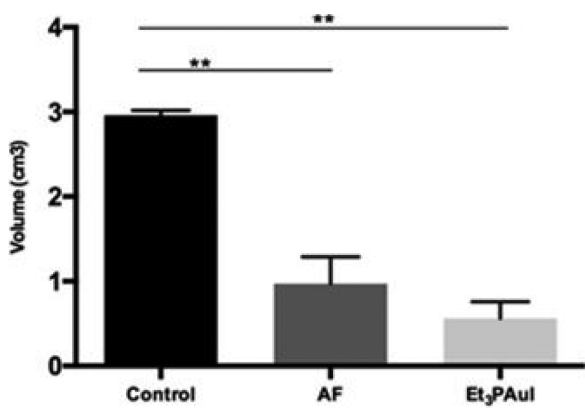

Figure 2. In vivo anticancer activity evaluation of gold compounds in a xenograft subcutaneous (sc) model of ovarian cancer. Mean volume of tumor masses obtained at the sacrifice, after 3 weeks from injection of A2780 cells. Data are reported as the mean $\pm \operatorname{SD}(* p<0.05, * * p<$ $0.01)$. Further details are reported in the Supporting Information.

$0,79 \pm 0,27, p<0.01)$. The above experimental protocol did not alter the behavior, vitality, or weight of treated mice.

Subsequently, the in vivo anticancer activity of $\mathbf{E t}_{3} \mathbf{P A u I}$ versus AF was assessed in an orthotopic model of ovarian cancer, a model that better represents the cancer behavior and response to therapy. Thirty female athymic nude mice were injected in the ovarian bursa $\left(5 \times 10^{5}\right.$ cells each mouse $)$ with A2780 cells transfected with the firefly luciferase gene (A2780luc). Autopsy confirmed that ovarian cancer occurred in all animals and representative images of the procedure and of tumor masses are reported in Figure 3A. The animals were divided in three groups of treatment (10 mice each): controls, AF treated and $\mathbf{E t}_{3} \mathbf{P A u I}$ treated. The administration schedule was the following: mice received compounds $(15 \mathrm{mg} / \mathrm{kg})$ ip three times a week for 2 weeks. The rationale for such a treatment schedule relied on the schedule reported by Mirabelli et al. ${ }^{5}$ and on the above-reported biodistribution data (Supporting Information) showing that administration of $15 \mathrm{mg} / \mathrm{kg}$ gives rise to a peak peripheral blood concentration of 823.19 and $944.17 \mathrm{ng} / \mathrm{mL}$, for $\mathbf{A F}$ and $\mathbf{E t}_{3} \mathbf{P A u I}$, respectively, after $24 \mathrm{~h}$. Mice were monitored daily, and the luciferase activity signal was quantified weekly (representative panels are reported in Figure 3B).

Both gold compounds effectively reduced the tumor burden (Figure $3 \mathrm{~B}$ ); notably, $\mathbf{E t}_{3} \mathbf{P A u I}$ is already very effective in antitumor activity after few doses (i.e., 1 week of treatment) leading to an almost complete tumor remission, as evidenced by the drastic reduction of the bioluminescent signal (ROI levels) compared to the control group (control: $20260 \pm 4631$ vs $\mathbf{E t}_{3}$ PAuI: $4729 \pm 2881 ; p<0,01$ ) (see graph in Figure 3C).

The tumor masses of the sacrificed animals after 3 weeks of treatment were collected and measured with caliper; the volume $\left(\mathrm{cm}^{3}\right)$ was calculated using the ellipsoid equation even for this model. ${ }^{21}$ Most important, the tumor masses of the animals treated with $\mathbf{E t}_{3}$ PAuI showed a very strong reduction of the volume compared both to the control group (control vs $\mathrm{Et}_{3}$ PAuI $p<0.05$ ) and to the AF group (AF: $6.86 \pm 1.57$ vs Et $_{3}$ PAuI: $1.00 \pm 0.70, p<0.01$ ) (Figure 3D). This latter evidence points out that $\mathbf{E t}_{3} \mathbf{P A u I}$ is a more effective anticancer drug compared with AF in our animal model.

In conclusion, we have shown here that a specific structural modification of the repurposed gold(I) drug AF, now under consideration in clinical trials for cancer treatment, i.e., the substitution of the thiosugar ligand with iodide, affords a distinct gold(I) complex $\mathbf{E t}_{3} \mathbf{P A u I}$, whose anticancer profile in vitro roughly resembles that of AF. This confirms that, even in
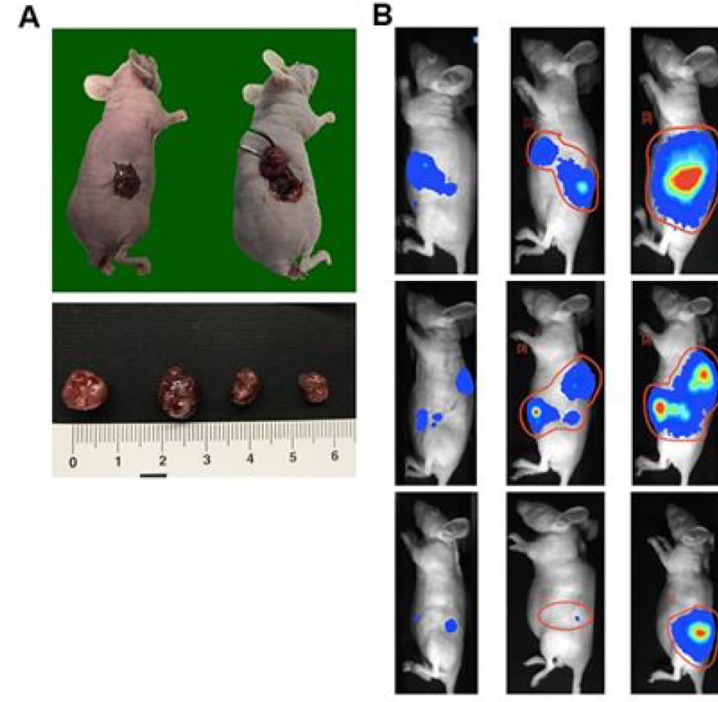

Control

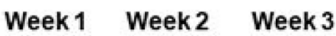

\section{AF}

Week 1 Week 2 Week 3
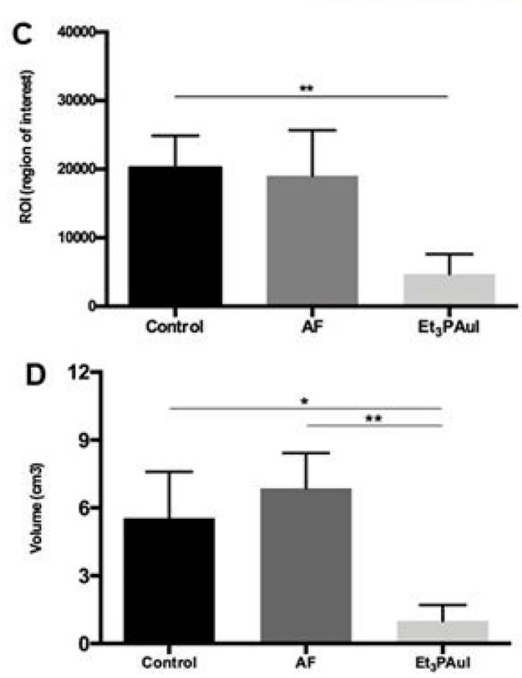

Figure 3. (A) Orthotopic model establishment: injection of $1 \times 106$ A2780-luc cells in the ovary bursa of nude mice. Representative macroscopic images of ovarian tumor masses obtained are showed in the bottom panels. (B) Representative pseudocolor BLI images tracking A2780-luc cells emission in mice, 7, 14, and 21 days after A2780-luc injection. Color bar represents light intensity levels reported as counts per minute (cpm). (C) Levels of ROI after 1 week of treatment; data are reported as mean of each group $(n=10$ animals/group) $\pm \mathrm{SD}(* * p<0.01)$. (D) Volume of tumor masses (mean $\pm \mathrm{SD})$ at the sacrifice after 3 weeks from injection of A2780luc cells (control group, $n=8$; AF, $n=5$; Et3PAuI, $n=5$ ). ${ }^{*} p<0.05$, $* * p<0.01$. Further details are reported in the Supporting Information.

the present ovarian cancer model, the thiosugar ligand is not mechanistically indispensable and that the $\left[\mathrm{Et}_{3} \mathrm{PAu}\right]^{+}$moiety is likely to be the true pharmacophore. Very interestingly, in our in vivo models, $\mathbf{E t}_{3} \mathbf{P A u I}$, has turned out to be far more effective than AF in contrasting tumor growth. Particularly impressive are the results and the differences between $\mathbf{A F}$ and $\mathbf{E t}_{3} \mathbf{P A u I}$ observed in the orthotopic model of ovarian cancer. $\mathbf{E t}_{\mathbf{3}} \mathbf{P A u I}$ is found to induce a very large tumor regression after only 1 week of treatment, as pointed out by the decrease of the bioluminescent signal. A conspicuous shrinkage of the tumor 
is still evident after 3 weeks. The enhanced anticancer activity observed in this orthotopic model might well arise from the far greater lipophilicity of $\mathbf{E t}_{3} \mathbf{P A u I}$, which might lead to a more favorable pharmacokinetics and biodistribution. Notably, the improved in vivo anticancer activity of $\mathbf{E t}_{3} \mathbf{P A u I}$ over $\mathrm{AF}$ does not bring about any increased systemic toxicity, making $\mathbf{E t}_{3}$ PAuI a promising new drug candidate. In view of its far superior efficacy in animal models, $\mathbf{E t}_{3} \mathbf{P A u I}$ merits a more extensive preclinical testing as it might be a valuable alternative to AF in cancer clinical trials.

\section{ASSOCIATED CONTENT}

\section{S Supporting Information}

The Supporting Information is available free of charge on the ACS Publications website at DOI: 10.1021/acsmedchemlett.9b00007.

Synthesis and purity of $\mathrm{Et}_{3} \mathrm{PAuI}, \log \mathrm{P}$ determinations, apoptosis plots; cell death and caspase activity; cell growth inhibition studies (sulforhodamine B assay); tolerability studies; biodistribution studies; in vivo anticancer activity evaluation xenograft subcutaneous models plots; NMR stability (PDF)

\section{AUTHOR INFORMATION}

\section{Corresponding Authors}

*E-mail: luigi.messori@unifi.it.

*E-mail: serena.pillozzi@unifi.it.

\section{ORCID}

Tiziano Marzo: 0000-0002-2567-3637

Alessandro Pratesi: 0000-0002-9553-9943

Francesca Magherini: 0000-0001-8388-0952

Tania Gamberi: 0000-0003-1489-8934

Luigi Messori: 0000-0002-9490-8014

\section{Author Contributions}

$\nabla_{\text {These authors contributed equally. The manuscript was }}$ written through contributions of all authors. All authors have given approval to the final version of the manuscript.

\section{Notes}

The authors declare no competing financial interest.

\section{ACKNOWLEDGMENTS}

This study was supported by contributions of Associazione Italiana per la Ricerca sul Cancro, Milan (AIRC-IG16049) and Istituto Toscano Tumori (Messori- ITT-2015) to L.Me. T.M. thanks AIRC-FIRC (Fondazione Italiana per la Ricerca sul Cancro, Milan) 3-years Fellowship for Italy (Project Code: 18044) and University of Pisa (PRA_2017_25). CIRCMSB and ente CRF are gratefully acknowledged. Animal experiments have been approved and authorized in accordance with the Italian law, reference code: 113/2016-PR 03/2016.

\section{REFERENCES}

(1) Bombardier, C.; Ware, J.; Russell, I. J.; Larson, M.; Chalmers, A.; Read, J. L. Auranofin therapy and quality of life in patients with rheumatoid arthritis. Results of a multicenter trial. Am. J. Med. 1986, $81,565-578$.

(2) Shaw, C. F. Gold-Based Therapeutic Agents. Chem. Rev. 1999, 99, 2589-2600.

(3) Cassetta, M. I.; Marzo, T.; Fallani, S.; Novelli, A.; Messori, L. Drug repositioning: auranofin as a prospective antimicrobial agent for the treatment of severe staphylococcal infections. BioMetals 2014, 27, 787-791.
(4) Capparelli, E. V.; Bricker-Ford, R.; Rogers, M. J.; McKerrow, J. H.; Reed, S. L. Phase I Clinical Trial Results of Auranofin, a Novel Antiparasitic Agent. Antimicrob. Agents Chemother. 2017, 61, e01947.

(5) Mirabelli, C. K.; Johnson, R. K.; Sung, C. M.; Faucette, L.; Muirhead, K.; Crooke, S. T. Evaluation of the in vivo antitumor activity and in vitro cytotoxic properties of auranofin, a coordinated gold compound, in murine tumor models. Cancer Res. 1985, 45, 3239.

(6) Simon, T. M.; Kunishima, D. H.; Vibert, G. J.; Lorber, A. Cellular antiproliferative action exerted by auranofin. J. Rheumatol. Suppl. 1979, 5, 91-97.

(7) Mirabell, C. K.; Johnson, R. K.; Hill, D. T.; Faucette, L. F.; Girard, G. R.; Kuo, G. Y.; Sung, C. M.; Crooke, S. T. Correlation of the in vitro cytotoxic and in vivo antitumor activities of gold(I) coordination complexes. J. Med. Chem. 1986, 29, 218-223.

(8) Hou, G.-X.; Pan-Pan, L.; Shengyi, Z.; Mengqi, Y.; Jianwei, L.; Jing, Y.; Yumin, H.; Wen-Qi, J.; Shijun, W.; Peng, H. Elimination of stem-like cancer cell side-population by auranofin through modulation of ROS and glycolysis. Cell Death Dis. 2018, 9, 89.

(9) Rios Perez, M. V.; Roife, D.; Dai, B. B.; Kang, Y.; Li, X.; Pratt, M.; Fleming, J. B. Auranofin to prevent progression of pancreatic ductal adenocarcinoma. J. Clin. Oncol. 2016, 34, 236.

(10) Roh, J. L.; Jang, H.; Kim, E. H.; Shin, D. Targeting of the Glutathione, Thioredoxin, and Nrf2 Antioxidant Systems in Head and Neck Cancer, Antioxid. Antioxid. Redox Signaling 2017, 27, 106-114.

(11) Auranofin in Treating Patients With Recurrent Epithelial Ovarian, Primary Peritoneal, or Fallopian Tube Cancer. https:// clinicaltrials.gov/ct2/show/NCT01747798.

(12) Sirolimus and Auranofin in Treating Patients With Advanced or Recurrent Non-Small Cell Lung Cancer or Small Cell Lung Cancer. https://clinicaltrials.gov/ct2/show/NCT01737502.

(13) Phase I and II Study of Auranofin in Chronic Lymphocytic Leukemia (CLL). https://clinicaltrials.gov/ct2/show/ NCT01419691.

(14) Scalcon, V.; Bindoli, A.; Rigobello, M. P. Significance of the mitochondrial thioredoxin reductase in cancer cells: An update on role, targets and inhibitors. Free Radical Biol. Med. 2018, 127, 62-79.

(15) Magherini, F.; Fiaschi, T.; Valocchia, E.; Becatti, M.; Pratesi, A.; Marzo, T.; Massai, L.; Gabbiani, C.; Landini, I.; Nobili, S.; Mini, E.; Messori, L.; Modesti, A.; Gamberi, T. Antiproliferative effects of two gold(I)-N-heterocyclic carbene complexes in A2780 human ovarian cancer cells: a comparative proteomic study. Oncotarget 2018, 9, 28042-28068.

(16) Pratesi, A.; Gabbiani, C.; Michelucci, E.; Ginanneschi, M.; Papini, A. M.; Rubbiani, R.; Ott, I.; Messori, L. Insights on the mechanism of thioredoxin reductase inhibition by Gold $\mathrm{N}$ heterocyclic carbene compounds using the synthetic linear Selenocysteine containing C-terminal peptide hTrxR(488-499): An ESI-MS investigation. J. Inorg. Biochem. 2014, 136, 161-169.

(17) Micale, N.; Schirmeister, T.; Ettari, R.; Cinellu, M. A.; Maiore, L.; Serratrice, M.; Gabbiani, C.; Massai, L.; Messori, L. Selected cytotoxic gold compounds cause significant inhibition of $20 \mathrm{~S}$ proteasome catalytic activities. J. Inorg. Biochem. 2014, 11, 79-82.

(18) Marzo, T.; Cirri, D.; Gabbiani, C.; Gamberi, T.; Magherini, F.; Pratesi, A.; Guerri, A.; Biver, T.; Binacchi, F.; Stefanini, M.; Arcangeli, A.; Messori, L. Auranofin, $\mathrm{Et}_{3} \mathrm{PAuCl}$, and $\mathrm{Et}_{3} \mathrm{PAuI}$ Are Highly Cytotoxic on Colorectal Cancer Cells: A Chemical and Biological Study. ACS Med. Chem. Lett. 2017, 8, 997-1001.

(19) Sutton, B. M.; Mc Gusty, E.; Walz, D. T.; Di Martino, M. J. Oral gold. Antiarthritic properties of alkylphosphinegold coordination complexes. J. Med. Chem. 1972, 15, 1095-1098.

(20) Marzo, T.; Cirri, D.; Pollini, S.; Prato, M.; Fallani, S.; Cassetta, M. I.; Novelli, A.; Rossolini, G. M.; Messori, L. Auranofin and its Analogues Show Potent Antimicrobial Activity Covering Multiresistant Pathogens: Structure-Activity Relationships. ChemMedChem 2018, 13, 2448-2454.

(21) Crociani, O.; Zanieri, F.; Pillozzi, S.; Lastraioli, E.; Stefanini, M.; Fiore, A.; Fortunato, A.; D’Amico, M.; Masselli, M.; De Lorenzo, E.; Gasparoli, L.; Chiu, M.; Bussolati, O.; Becchetti, A.; Arcangeli, A. 
hERG1 channels modulate integrin signaling to trigger angiogenesis and tumor progression in colorectal cancer. Sci. Rep. 2013, 3, 3308. 